Research Article

\title{
Fractals via Generalized Jungck-S Iterative Scheme
}

\author{
Zhihua Chen, ${ }^{1}$ Muhammad Tanveer $\mathbb{D D}^{2}$ Waqas Nazeer, ${ }^{3}$ and Jing Wu $\mathbb{D}^{4}$ \\ ${ }^{1}$ Institute of Computing Science and Technology, Guangzhou University, Guangzhou 510006, China \\ ${ }^{2}$ Department of Mathematics and Statistics, The University of Lahore, Lahore, Pakistan \\ ${ }^{3}$ Department of Mathematics, GC University, Lahore, Pakistan \\ ${ }^{4}$ School of Science, Xijing University, Xian 710123, China \\ Correspondence should be addressed to Jing Wu; specialwujing@sina.com
}

Received 8 September 2020; Revised 24 September 2020; Accepted 9 February 2021; Published 1 March 2021

Academic Editor: Paolo Renna

Copyright (c) 2021 Zhihua Chen et al. This is an open access article distributed under the Creative Commons Attribution License, which permits unrestricted use, distribution, and reproduction in any medium, provided the original work is properly cited.

\begin{abstract}
The purpose of this research is to introduce a Jungck-S iterative method with $\left(m, h_{1}, h_{2}\right)$-convexity and hence unify different comparable iterative schemes existing in the literature. A Jungck-S orbit is constructed, and escape radius is derived with our scheme. A new escape radius is also obtained for generating the fractals. Julia and Mandelbrot set are visualized with the help of proposed algorithms based on our iterative scheme. Moreover, we present some complex graphs of Julia and Mandelbrot sets using the derived orbit and discuss their nature in detail.
\end{abstract}

\section{Introduction}

The word fractal originating from Latin language means to divide or break. This is tantamount to self-similar patterns in the complex graphics. The fractal theory has many applications in mathematics and different related disciplines. In biological sciences, this theory is being applied successfully to understand certain biologic phenomena, for example, growth culture for the microorganism, like bacteria or amoeba, and to study and analyze the fibre pattern of nerve and so forth. In physical sciences, fractals are used to determine and understand the turbulent flows in fluid mechanics. In telecommunication, fractals are used to manufacture antennae. Moreover, computer networking, radar system, and architectural models also fall into the domain of applications of fractals theory.

In 1975, Mandelbrot generated a sequence of iterates for a complex polynomial $z^{2}+c$ [1] under some restrictions. He observed a chain of self-similar patterns on a graph and hence named it fractal. He claimed that the obtained image was the sequence of connected Julia sets. After his valuable work, the researcher generalized the fractals in many different ways. It is worth mentioning that complex graphical shapes, fractals, were discovered as the fixed points of certain set maps. So, the fixed point theory plays a vital role in the investigation of fractals. Different iterative schemes, mainly used to approximate the fixed points of certain mappings, can be employed to sketch some beautiful natural scenes of sunset, lighting, rainbow, galaxies, deserts, mountains, and so forth. These aesthetic patterns depend on the iterative algorithms and hence provide a good source of motivation to apply mathematical models in art and designs. The generalized form of the Mandelbrot set was given in [2].

Some logarithmic, rational, exponential, and trigonometric functions were investigated in [3]. The bicomplex, tricomplex, and quaternions functions were utilized in [4-6] to create some generalized versions of fractals. The study of fractals using fixed point theory attracted the attention of several researchers after the work of Rani et al. in $[7,8]$. Some types of fractals via different explicit iterations were analyzed in [9-13].

In this paper, we define the orbit of generalized Jungck-S iterative scheme with $\left(m, h_{1}, h_{2}\right)$-convexity for fractals. We derive a new escape radius for complex polynomials and develop the algorithms for fractals visualization and draw some complex graphs of Julia and Mandelbrot sets using computer software. The paper is organized as follows: in Section 2, some known concepts and iterative schemes are 
given. Section 3 contains the Jungck-S orbit with $\left(m, h_{1}, h_{2}\right)$-convexity (JSOmhh) and the derivation of escape radius for Jungck-S iterative scheme with $\left(m, h_{1}, h_{2}\right)$-convexity for general complex polynomial. In Section 4 , we visualize some quadratic and cubic complex graphs of Julia and Mandelbrot sets. Characteristics of Julia and Mandelbrot sets are also discussed in this section. In Section 5, the summary of our present work and the plan of our future work are presented.

\section{Preliminaries}

Consistent with $[14,15]$, the following definitions will be needed in the sequel.

Definition 1 (see [14]). Let $f: \mathbb{C} \longrightarrow \mathbb{C}$ be a complex polynomial of degree $\geq 2$ with complex coefficients and $f^{p}(x)$ the $p^{\text {th }}$ iterate of $x$. The behavior of the iterate $f^{p}(x)$ for large $p$ determines the Julia set. The set $F_{f}$, called filled Julia set, is the set of all those points of $\mathbb{C}$ for which the orbits $\left.\left|f^{p}(z)\right|\right\}_{p=0}^{\infty} \nrightarrow \infty$ are as $p \longrightarrow \infty$; that is,

$$
F_{f}=\left\{z \in \mathbb{C}:\left\{\left|f^{p}(z)\right|\right\}_{p=0}^{\infty} \text { is bounded }\right\} .
$$

The boundary of the filled Julia set is called simply Julia set.

Mandelbrot [15] extended the concept of a Julia set and presented the notion of fractals. He investigated the graphical behavior of connected Julia sets and plotted them for complex function, $f_{c}(x)=x^{2}+c$, where $x \in \mathbb{C}$ is a variable and $c \in \mathbb{C}$ is the input.

Definition 2 (see [15]). The set which consists of all those points (parameters) of $\mathbb{C}$ for which the Julia sets are connected is called Mandelbrot set $M$; that is,

$$
M=\left\{c \in \mathbb{C}: F_{f} \text { is connected }\right\} .
$$

Equivalently, the Mandelbrot set can be defined as follows [16]:

$$
M=\left\{c \in \mathbb{C}:\left\{f^{p}(0)\right\} \text { does not tend to } \infty \text { as } p \longrightarrow \infty\right\} .
$$

Different algorithms have been employed to generate Julia sets. Some popular algorithms to visualize the Julia sets are distance estimator [17], escape time [18], and potential function algorithms [19]. The escape time algorithms have been used in this paper.

We established escape time algorithms, namely, Algorithms 1 and 2 with derived escape radius to generate the fractals.

Let us refer to some iterative algorithms: the Jungck iteration was studied in [20], the Jungck-Mann iteration with $s$-convexity was studied in [21], the Jungck-Ishikawa iteration with $s$-convexity was studied in [21], the following Jungck-S iteration was studied in [20] and Kwun et al.[22] used a convex combination to define the Jungck-S iterative scheme with $s$-convexity. We define the Jungck-S iterative scheme with $\left(m, h_{1}, h_{2}\right)$-convexity (JSOmhh) in the following manner:

Definition 3 (JSOmhh). Suppose that $P, Q: \mathbb{C} \longrightarrow \mathbb{C}$, where $P$ is one to one. Let $z_{0} \in \mathbb{C}$ be an initial point. The Jungck-S iterative scheme with $\left(m, h_{1}, h_{2}\right)$-convexity is defined as follows:

$$
\left\{\begin{array}{l}
P\left(z_{k+1}\right)=m h_{1}(\alpha) Q\left(z_{k}\right)+h_{2}(\alpha) Q\left(y_{k}\right), \\
P\left(y_{k}\right)=m h_{1}(\beta) P\left(z_{k}\right)+h_{2}(\beta) Q\left(z_{k}\right),
\end{array}\right.
$$

where $\quad h_{1}(t)=(1-t)^{-s}$ and $\quad h_{2}(t)=t^{-s}$, also $\alpha, \beta, s, m \in(0,1]$, and $k=0,1,2, \ldots$

It is important to mention here that our scheme generalizes many of the above-mentioned schemes.

\section{Main Results}

In this section, we first introduce the Jungck-S iterative scheme with $\left(m, h_{1}, h_{2}\right)$-convexity (JSOmhh) and then prove escape criteria to determine the escape radius induced by (JSOmhh).

In the following theorem, we use (JSOmhh) to establish the escape criteria for $f(z)=\sum_{i=0}^{p} a_{i} z^{i}$, where $p \geq 2, a_{i} \in \mathbb{C}$, for $i=0,1,2, \ldots, p$ and $\left|a_{p}\right|>\sum_{i=2}^{p-1}\left|a_{i}\right|$ with choices $Q(z)=$ $\sum_{i=2}^{p} a_{i} z^{i}+a_{0}$ and $P(z)=a_{1} z$ to generate fractals.

Theorem 1. Suppose that $|z| \geq\left|a_{0}\right|>\left(2\left(1+m\left|a_{1}\right|\right) /\right.$ $s \alpha(\phi-\varphi))^{1 /(p-1)}$ and $|z| \geq\left|a_{0}\right|>\left(2\left(1+m\left|a_{1}\right|\right) / s \beta\right.$ $(\phi-\varphi))^{1 /(p-1)} \quad$ where $\phi=\left|a_{p}\right|, \varphi=\sum_{2}^{p-1}\left|a_{i}\right|$, where $\alpha, \beta, s \in(0,1]$, and $\left\{z_{k}\right\}_{k \in \mathbb{N}}$ is defined as

$$
\left\{\begin{array}{l}
P\left(z_{k+1}\right)=m h_{1}(\alpha) P\left(z_{k}\right)+h_{2}(\alpha) Q\left(y_{k}\right), \\
P\left(y_{k}\right)=m h_{1}(\beta) P\left(z_{k}\right)+h_{1}(\beta) Q\left(z_{k}\right),
\end{array}\right.
$$

where $s, t, m \in(0,1], h_{1}(t)=(1-t)^{-s}, h_{2}(t)=t^{-s} \quad$ and $k=0,1,2, \ldots$. Then, $\left|z_{k}\right| \longrightarrow \infty$ as $k \longrightarrow \infty$.

Proof. Since $f(z)=\sum_{i=0}^{p} a_{i} z^{i}$, where $a_{i} \in \mathbb{C}$ for $i=0,1,2, \ldots, p, z_{0}=z$, and $y_{0}=y$. Handling $f$ as $f=Q-$ $P$ with choice $Q(z)=\sum_{i=2}^{p} a_{i} z^{i}+a_{0}$ and $P(z)=a_{1} z$, then

$$
\begin{aligned}
\left|P\left(y_{0}\right)\right| & =\left|m h_{1}(\beta) P(z)+h_{2}(\beta) Q(z)\right| \\
& =\left|m(1-\beta)^{-s} a_{1} z+\beta^{-s}\left(\sum_{i=2}^{p} a_{i} z^{i}+a_{0}\right)\right|\left|a_{1} y_{0}\right| \\
& \geq\left|s \beta\left(\sum_{i=2}^{p} a_{i} z^{i}+a_{0}\right)\right|-\left|m a_{1} z\right| .
\end{aligned}
$$

Because $(1-\beta)^{-s}=1+\beta s+\cdots>1$ and $\beta^{-s}=1+s(1-$ $\beta)+\cdots>\beta s$ for all $s, m, \beta \in(0,1]$, therefore

$$
\left|a_{1} y_{0}\right| \geq\left|s \beta\left(\sum_{i=2}^{p} a_{i} z^{i}+a_{0}\right)\right|-s \beta\left|a_{0}\right|-\left|m a_{1} z\right| \text {. }
$$

Since $|z| \geq\left|a_{0}\right|$ and $s \beta<1$, we have 
Input: $f(z)=\sum_{i=0}^{p} a_{i} z^{i}$ where $p \geq 2, a_{i} \in \mathbb{C}$ for $i=0,1,2, \ldots, p$-polynomial of complex variable, $\phi, \varphi, \alpha, \beta, s, m-$ involved parameters, $A=\left[x_{\min }, x_{\max }\right] \times\left[y_{\min }, y_{\max }\right]$-occupied area, $K$ - fixed number of iterates, colourfunction [0..n-1]-colour function with $n$ colours.

Output: a Julia set.

(1) for $a_{0} \in \mathbb{C}$ do

(2) $\quad R=\operatorname{Max}\left[\left|a_{0}\right|,\left(2\left(1+m\left|d_{1}\right|\right) / s \alpha(\phi-\varphi)\right)^{1 /(p-1)},\left(2\left(1+m\left|d_{1}\right|\right) / s \beta(\phi-\varphi)\right)^{1 /(p-1)}\right]$ the escape radius

(3) $k=0$

(4) $z_{0}=a_{0}$-initial guess for $f$

(5) while $k \leq K$ do

(6) $z_{k+1}=f_{c}\left(z_{k}\right)$

(7) if $\left|z_{k+1}\right|>R$ then

(8) break

(9) $\quad k=k+1$

(10) $\quad c=\lfloor(n-1)(k / K)\rfloor$

(11) colour $z_{0}$ with colourfunction $[c]$.

Algorithm 1: Complex graph of Julia set.

Input: $f(z)=\sum_{i=0}^{p} a_{i} z^{i}$ where $p \geq 2, a_{i} \in \mathbb{C}$ for $i=0,1,2, \ldots, p$ - polynomial of complex variable, $\phi, \varphi, \alpha, \beta, s, m-$ involved parameters, $A=\left[x_{\min }, x_{\max }\right] \times\left[y_{\min }, y_{\max }\right]$-occupied area, $K$ - fixed number of iterates, colourfunction [0..n-1]-colour function with $n$ colours.

Output: a Mandelbrot set.

(1) for $a_{0} \in \mathbb{C}$ do

(2) $\quad R=\operatorname{Max}\left[\left|a_{0}\right|,\left(2\left(1+m\left|d_{1}\right|\right) / s \alpha(\phi-\varphi)\right)^{1 /(p-1)},\left(2\left(1+m\left|d_{1}\right|\right) / s \beta(\phi-\varphi)\right)^{1 /(p-1)}\right]-$ the escape radius

(3) $k=0$

(4) $\quad z_{0}=0$-initial guess for $f$

(5) while $k \leq K$ do

(6) $z_{k+1}=f\left(z_{k}\right)$

(7) if $\left|z_{k+1}\right|>R$ then

(8) break

(9) $k=k+1$

(10) $\quad c=\lfloor(n-1)(k / K)\rfloor$

(11) colour $a_{0}$ with colourfunction $[c]$.

Algorithm 2: Complex graph of Mandelbrot.

$$
\begin{aligned}
\left|a_{1} y_{0}\right| & \geq s \beta\left|\sum_{i=2}^{p} a_{i} z^{i}\right|-|z|-m\left|a_{1}\right||z| \\
& =|z|\left(s \beta\left|\sum_{i=2}^{p} a_{i} z^{i-1}\right|-\left(1+m\left|a_{1}\right|\right)\right)\left|y_{0}\right| \\
& =\frac{1}{\left|a_{1}\right|}\left\{|z|\left(s \beta\left|\sum_{i=2}^{p} a_{i} z^{i-1}\right|-\left(1+m\left|a_{1}\right|\right)\right)\right\} .
\end{aligned}
$$

Since $\left(1+m\left|a_{1}\right|\right)>\left|a_{1}\right|$, then

$$
\begin{aligned}
\left|y_{0}\right| & \geq|z|\left(\frac{s \beta\left|\sum_{i=2}^{p} a_{i} z^{i-1}\right|}{1+m\left|a_{1}\right|}-1\right) \\
& \geq|z|\left(\frac{s \beta\left|z^{p-1}\right|\left(\left|a_{p}\right|-\sum_{i=2}^{p-1}\left|a_{i}\right|\right)}{1+m\left|a_{1}\right|}-1\right) \\
& =|z|\left(\frac{s \beta\left|z^{p-1}\right|(\phi-\varphi)}{1+m\left|a_{1}\right|}-1\right)\left|y_{0}\right| \\
& \geq s \beta|z| .
\end{aligned}
$$

Because $\quad|z| \geq\left|a_{0}\right|>\left(2\left(1+m\left|a_{1}\right|\right) / s \beta(\phi-\varphi)\right)^{1 /(p-1)}$ where $\phi=\left|a_{p}\right|, \varphi=\sum_{2}^{p-1}\left|a_{i}\right|$, this produced the situation $|z|\left(\left(|z|^{p-1}(s \beta(\phi-\varphi)) /\left(1+m\left|a_{1}\right|\right)\right)-1\right)>|z| \geq s \beta|z|$. For the last step, we have

$$
\begin{aligned}
\left|P\left(z_{1}\right)\right| & =\left|m h_{1}(\alpha) Q\left(z_{0}\right)+h_{2}(\alpha) Q\left(y_{0}\right)\right|\left|a_{1} z_{1}\right| \\
& =\left|m(1-\alpha)^{-s}\left(z^{p}+d_{2}\right)+\alpha^{-s}\left(y^{p}+d_{2}\right)\right| \\
& \geq\left|m\left(\sum_{i=2}^{p} a_{i} z^{i}+a_{0}\right)+s \alpha\left(\sum_{i=2}^{p} a_{i} y^{i}+a_{0}\right)\right| .
\end{aligned}
$$

Since $m>0$, then we get

$$
\begin{aligned}
\left|a_{1} z_{1}\right| & \geq s \alpha\left|\sum_{i=2}^{p} a_{i} y^{i}+a_{0}\right| \\
& \geq s \alpha\left|\sum_{i=2}^{p} a_{i} y^{i}\right|-\left|a_{0}\right| \\
& \geq|z|\left\{s^{2} \alpha \beta\left|z^{p-1}\right|\left(\left|a_{p}\right|-\sum_{i=2}^{p-1}\left|a_{i}\right|\right)-1\right\} .
\end{aligned}
$$


Hence,

$$
\left|z_{1}\right| \geq|z|\left(\frac{s^{2} \alpha \beta\left|z^{p-1}\right|(\phi-\varphi)}{1+m\left|a_{1}\right|}-1\right) .
$$

Since $|z|>\left(2\left(1+m\left|a_{1}\right|\right) / s \alpha(\phi-\varphi)\right)^{1 /(p-1)}$ and $|z|>$ $\left(2\left(1+m\left|a_{1}\right|\right) / s \beta(\phi-\varphi)\right)^{1 /(p-1)}$, so $|z|^{p-1}>2\left(1+m\left|d_{1}\right|\right)$ $/ s^{2} \alpha \beta$ and this implies $\left(\left(s^{2} \alpha \beta(\phi-\varphi)|z|^{p-1}\right) /(1+m\right.$ $\left.\left.\left|d_{1}\right|\right)\right)-1>1$. Therefore, there exists $\delta>0$ such that $\left(s^{2} \alpha \beta(\phi-\varphi)|z|^{p-1} /\left(1+m\left|d_{1}\right|\right)\right)-1>1+\delta$. Consequently, $\left|z_{1}\right|>(1+\delta)|z|$. In particular, $\left|z_{1}\right|>|z|$. So, we may iterate to find $\left|z_{i}\right|>(1+\delta)^{i}|z|$. Hence, the orbit of $z$ tends to infinity and this completes the proof.

Theorem 2. Suppose that $\left\{z_{i}\right\}_{i \in \mathbb{N}}$ is a sequence of points in JSOmhh for complex polynomial $f(z)=\sum_{i=0}^{p} a_{i} z^{i}$, where $p \geq 2, a_{i} \in \mathbb{C}$, for $i=0,1,2, \ldots, p, \phi=\left|a_{p}\right|$, and $\varphi=\sum_{i=2}^{p-1}\left|a_{i}\right|$ with $\phi>\varphi$ such that $\left|z_{i}\right| \longrightarrow \infty$ as $i \longrightarrow \infty$; then $|z| \geq\left|a_{0}\right|>\left(2\left(1+m\left|d_{1}\right|\right) / s \alpha(\phi-\varphi)\right)^{1 /(p-1)}$ and $|z| \geq\left|a_{0}\right|>$ $\left(2\left(1+m\left|d_{1}\right|\right) / s \beta(\phi-\varphi)\right)^{1 /\left(p^{-1}\right)}$ where $\alpha, \beta, s \in(0,1]$.

Proof. Since $\left\{z_{i}\right\}_{i \in \mathbb{N}}$ is the sequence of points in JSOmhh for complex polynomial $f(z)=\sum_{i=0}^{p} a_{i} z^{i}$ where $p \geq 2, a_{i} \in \mathbb{C}$ for $i=0,1,2, \ldots, p, \phi=\left|a_{p}\right|$ and $\varphi=\sum_{i=2}^{p-1}\left|a_{i}\right|$ with $\phi>\varphi$ such that $\left|z_{i}\right| \longrightarrow \infty$ as $i \longrightarrow \infty$, therefore there exists $\delta>0$ such that

$$
\left|z_{i}\right|>(1+\delta)^{i}|z|
$$

For $i=1$, we get

$$
\left|z_{1}\right| \geq(1+\delta)|z|
$$

Since $f(z)=\sum_{i=0}^{p} a_{i} z^{i}$, where $a_{i} \in \mathbb{C}$ for $i=0,1,2, \ldots, p$, $z_{0}=z$ and $y_{0}=y$. Handling $f$ as $f=Q-P$ with choice $Q(z)=\sum_{i=2}^{p} a_{i} z^{i}+a_{0}$ and $P(z)=a_{1} z$, then

$$
\begin{aligned}
\left|P\left(y_{0}\right)\right| & =\left|m h_{1}(\beta) P(z)+h_{2}(\beta) Q(z)\right| \\
& =\left|m(1-\beta)^{-s} a_{1} z+\beta^{-s}\left(\sum_{i=2}^{p} a_{i} z^{i}+a_{0}\right)\right|\left|a_{1} y_{0}\right| \\
& \geq\left|s \beta\left(\sum_{i=2}^{p} a_{i} z^{i}+a_{0}\right)\right|-\left|m a_{1} z\right| .
\end{aligned}
$$

Because $(1-\beta)^{-s}=1+\beta s+\cdots>1$ and $\beta^{-s}=1+s(1-$ $\beta)+\cdots>\beta$ s for all $s, m, \beta \in(0,1]$, therefore

$$
\left|a_{1} y_{0}\right| \geq\left|s \beta\left(\sum_{i=2}^{p} a_{i} z^{i}+a_{0}\right)\right|-s \beta\left|a_{0}\right|-\left|m a_{1} z\right| .
$$

Since for fractal generation it must be true $|z| \geq\left|a_{0}\right|$. Also, $s \beta<1$, and we obtain

$$
\begin{aligned}
\left|a_{1} y_{0}\right| & \geq s \beta\left|\sum_{i=2}^{p} a_{i} z^{i}\right|-|z|-m\left|a_{1}\right||z| \\
& =|z|\left(s \beta\left|\sum_{i=2}^{p} a_{i} z^{i-1}\right|-\left(1+m\left|a_{1}\right|\right)\right)\left|y_{0}\right| \\
& =\frac{1}{\left|a_{1}\right|}\left\{|z|\left(s \beta\left|\sum_{i=2}^{p} a_{i} z^{i-1}\right|-\left(1+m\left|a_{1}\right|\right)\right)\right\} .
\end{aligned}
$$

Since $\left(1+m\left|a_{1}\right|\right)>\left|a_{1}\right|$, then

$$
\begin{aligned}
\left|y_{0}\right| & \geq|z|\left(\frac{s \beta\left|\sum_{i=2}^{p} a_{i} z^{i-1}\right|}{1+m\left|a_{1}\right|}-1\right) \geq|z|\left(\frac{s \beta\left|z^{p-1}\right|\left(\left|a_{p}\right|-\sum_{i=2}^{p-1}\left|a_{i}\right|\right)}{1+m\left|a_{1}\right|}-1\right) \\
& =|z|\left(\frac{s \beta\left|z^{p-1}\right|(\phi-\varphi)}{1+m\left|a_{1}\right|}-1\right)\left|y_{0}\right| \\
& \geq s \beta|z| .
\end{aligned}
$$

Since fractals are bounded, therefore $\left(\left(s b\left|z^{p-1}\right| /\left(1+\left|d_{1}\right|\right)\right)-1\right) \geq 1$. For the last step, we have

$$
\begin{aligned}
\left|P\left(z_{1}\right)\right| & =\left|m h_{1}(\alpha) Q\left(z_{0}\right)+h_{2}(\alpha) Q\left(y_{0}\right)\right|\left|a_{1} z_{1}\right| \\
& =\left|m(1-\alpha)^{-s}\left(z^{p}+d_{2}\right)+\alpha^{-s}\left(y^{p}+d_{2}\right)\right| \\
& \geq\left|m\left(\sum_{i=2}^{p} a_{i} z^{i}+a_{0}\right)+s a\left(\sum_{i=2}^{p} a_{i} y^{i}+a_{0}\right)\right| .
\end{aligned}
$$

Since $m>0$, then we get

$$
\begin{aligned}
\left|a_{1} z_{1}\right| & \geq s \alpha\left|\sum_{i=2}^{p} a_{i} y^{i}+a_{0}\right| \\
& \geq s \alpha\left|\sum_{i=2}^{p} a_{i} y^{i}\right|-\left|a_{0}\right| \\
& \geq|z|\left\{s^{2} \alpha \beta\left|z^{p-1}\right|\left(\left|a_{p}\right|-\sum_{i=2}^{p-1}\left|a_{i}\right|\right)-1\right\} .
\end{aligned}
$$

Hence, 


$$
\left|z_{1}\right| \geq|z|\left(\frac{s^{2} \alpha \beta\left|z^{p-1}\right|(\phi-\varphi)}{1+m\left|a_{1}\right|}-1\right) .
$$

Comparing (14) and (21), we have

$$
\begin{aligned}
\frac{s^{2} \alpha \beta(\phi-\varphi)\left|z^{p-1}\right|}{1+m\left|a_{1}\right|}-1 & =1+\delta \frac{s^{2} \alpha \beta(\phi-\varphi)\left|z^{p-1}\right|}{1+m\left|a_{1}\right|}-1 \\
& >1,
\end{aligned}
$$

because $\delta>0$. This implies

$$
|z|>\left(\frac{2\left(1+m\left|a_{1}\right|\right)}{s^{2} \alpha \beta(\phi-\varphi)}\right)^{1 /(p-1)} .
$$

As a result, we obtain $|z|>\left(2\left(1+m\left|a_{1}\right|\right)\right.$ $/ s \alpha(\phi-\varphi))^{1 /(p-1)}$ and $|z|>\left(2\left(1+m\left|a_{1}\right|\right) / s \beta(\phi-\varphi)\right)^{1 /(p-1)}$ where $p \geq 2$ and $\alpha, \beta, m, s \in(0,1]$. To visualize complex fractal, $|z| \geq\left|a_{0}\right|$ must exist because, for any given point $|z|<\left|a_{0}\right|$, we have to compute the JSOmhh of $z$. If, for some $i$, $\left|z_{i}\right|$ lies outside the circle of radius $\max \left\{\left|a_{0}\right|,\left(2\left(1+m\left|a_{1}\right|\right) /\right.\right.$ $\left.s \alpha(\phi-\varphi))^{1 /(p-1)},\left(2\left(1+m\left|a_{1}\right|\right) / s \beta(\phi-\varphi)\right)^{1 /(p-1)}\right\}$, we observed that JSOmhh escapes. Hence, $z$ is neither in Julia sets nor in Mandelbrot sets, but $\left|z_{i}\right|$ is bounded to obey $|z| \geq\left|a_{0}\right|$; then $\left|z_{i}\right|$ lies in JSOmhh. Hence, it is the desired result.

\section{Application of Fractals}

In this section, we use our developed algorithms and derived escape radius for JSOmhh to draw some attractive and inspiring Julia and Mandelbrot sets using the framework of Mathematica. Throughout this section, we use $K=100$ (for maximum iterates).

4.1. Julia Sets. Figures 1-6 are quadratic Julia sets at different involved parameters. We obverse that each graph of quadratic Julia set is different from the other. We use the same complex polynomial and involved parameters except for $a_{0}$ for Figures 1 and 2 and analyze that due to a very small change in $a_{0}$, the nature of graphs changes drastically. Also, for Figures 3-6, we have the same arguments. We also observe that the correspondence between the points of quadratic Julia sets creates the artful patterns and self-similarity in points of quadratic Julia sets show that the drawn graphs are the fractals. The involved parameters for complex graphs of quadratic Julia sets 1-6 were as follows:

Figure 1: $p=2, a_{0}=0.0248+0.0084 \mathbf{i}, a_{1}=1 / 2, a_{2}=2$, $a, b, s, m=0.1, A=[-0.27,0.27] \times[-0.37,0.37]$.

Figure 2: $p=2, a_{0}=-0.084, a_{1}=1 / 2, a_{2}=2, a, b, s$, $m=0.1, A=[-0.37,0.37] \times[-0.2,0.2]$.

Figure 3: $p=2, a_{0}=0.0015+0.00007 \mathbf{i}, a_{1}=1 / 2, a_{2}=$ $100, a, b, s, m=0.5, \quad A=[-0.007,0.005] \times[-0.0025$, 0.0025].
Figure 4: $p=2, a_{0}=0.0015+0.00001 \mathbf{i}, a_{1}=1 / 2, a_{2}=$ $100, a, b, s, m=0.5, \quad A=[-0.007,0.005] \times[-0.0025$, $0.0025]$.

Figure 5: $p=2, a_{0}=0.0071+0.0014 \mathbf{i}, a_{1}=2, a_{2}=1$, $a, b, s, m=0.9, A=[-0.053,0.053] \times[-0.035,0.035]$.

Figure 6: $p=2, a_{0}=0.006 \mathbf{i}, a_{1}=2, a_{2}=1, a, b, s, m=$ $0.9, A=[-0.053,0.053] \times[-0.037,0.037]$.

In Figures 7-12, cubic Julia sets are presented. Again, we use the same complex cubic polynomial and involved parameters except for $a_{0}$ for Figures 7 and 8 . We observe that, due to a very small change in $a_{0}$, the nature of cubic Julia graphs also changes drastically. We have made the same augments for Figures 9-12, respectively. Furthermore, we observe that the correspondence between the points of cubic Julia sets creates the aesthetic patterns and self-similarity in points of cubic Julia sets show that the drawn graphs of cubic Julia sets are also fractals. The involved parameters for complex graphs of cubic Julia sets 7-12 were as follows:

Figure 7: $p=3, a_{0}=0.00122, a_{1}=1+\mathbf{i}, a_{2}=1, a_{3}=$ $50, a, b, s, m=0.9, A=[-0.01,0.01] \times[-0.01,0.01]$.

Figure 8: $p=3, a_{0}=0.001019, a_{1}=1+\mathbf{i}, a_{2}=1, a_{3}=$ $50, a, b, s, m=0.9, A=[-0.012,0.01] \times[-0.012,0.01]$.

Figure 9: $p=3, a_{0}=0.000085 \mathbf{i}, a_{1}=1+\mathbf{i}, a_{2}=45, a_{3}=$ 50, $\quad a, b, s, m=0.9, \quad A=[-0.00007,0.00007] \times$ [-0.00007, 0.00007].

Figure 10: $p=3, a_{1}=1+\mathbf{i}, a_{2}=45, a_{3}=50, a, b, s, m$ $=0.9, A=[-0.00009,0.00009] \times[-0.00004,0.00004]$.

Figure 11: $p=3, a_{0}=0.09956+0.27 i, a_{1}=2, a_{2}=1$, $a_{3}=3, \quad a=0.1, b=0.9, s, m=0.5, \quad A=[-0.8,0.6] \times$ $[-0.6,0.7]$.

Figure 12: $p=3, a_{0}=0.0489+0.366 \mathbf{i}, a_{1}=2, a_{2}=1$, $a_{3}=3, \quad a=0.1, b=0.9, s, m=0.5, \quad A=[-0.8,0.6] \times$ $[-0.6,0.7]$.

4.2. Mandelbrot Sets. Figures 13-24 execute the quadratic complex polynomial for different involved parameters in JSOmhh. Figures 13-16 represent the quadratic Mandelbrot sets. Each quadratic Mandelbrot set has a main cardioid type body, one large bulb, and two small bulbs are seen on cardioid type body. The bulbs are self-similar and contain a large number of more small bulbs. Due to a small change in the involved parameters, the size of bulbs and main cardioid changes. Figures 17 and 18 are Mandelbrot sets for quadratic polynomial, but both figures are not like the usual quadratic Mandelbrot set. Some parts of both images are like the quadratic, cubic, and quadric Mandelbrot sets. It is observed that the values of $a_{i}$ 's are in the inverse proportion to the area $A$; that is, the larger the values of $a_{i}$ 's, the smaller the area $A$. The involved parameters for quadratic Mandelbrot sets were taken as follows:

Figure 13: $p=2, a_{1}=1 / 2, a_{2}=2, \quad a, b, s, m=0.1$, $A=[-0.17,0.05] \times[-0.1,0.1]$. 


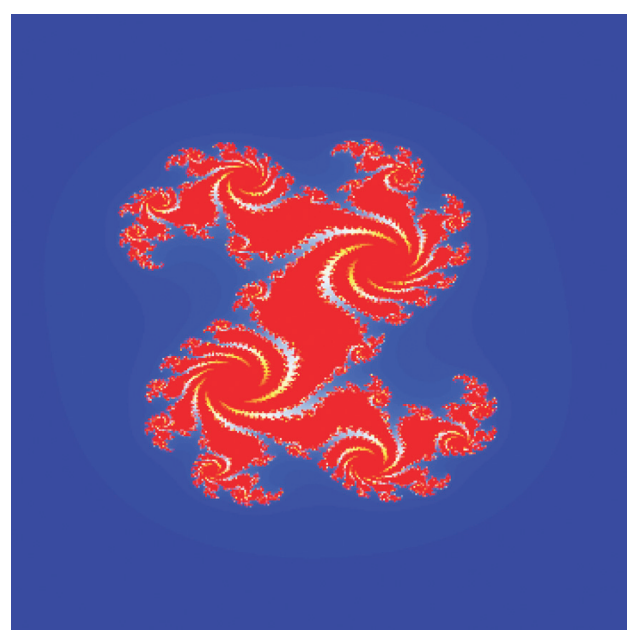

FIGURE 1: Quadratic Julia set in JSOmhh.

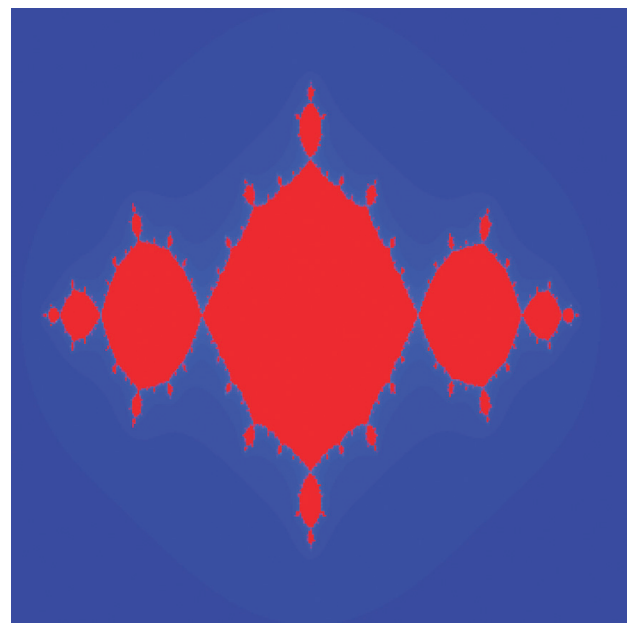

FIgURE 2: Quadratic Julia set in JSOmhh.

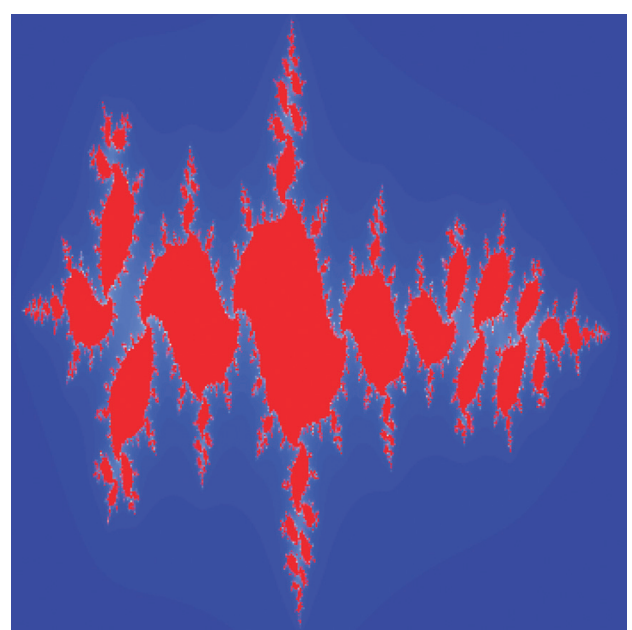

FIGURE 3: Quadratic Julia set in JSOmhh.



FIgURE 4: Quadratic Julia set in JSOmhh.

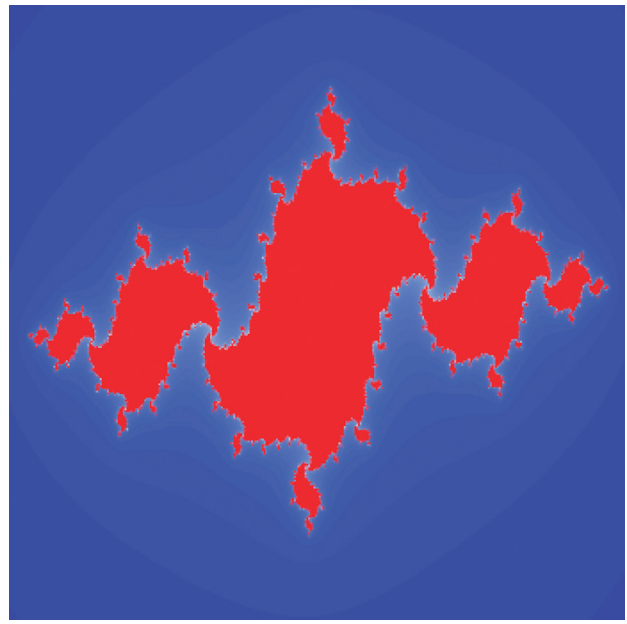

FIgURE 5: Quadratic Julia set in JSOmhh.

Figure 14: $\quad p=2, \quad a_{1}=3, a_{2}=10, \quad a, m=0.5$, $b=0.9, s=0.8, A=[-0.17,0.05] \times[-0.07,0.07]$.

Figure 15: $p=2, a_{1}=2, a_{2}=100, a=0.5, b=0.9$, $s=0.8, m=0.9, \quad A=[-0.0081,0.0005] \times[-0.001$, 0.001].

Figure 16: $p=2, a_{1}=100, a_{2}=1, a, b=0.5, s=0.9$, $m=0.7, A=[-4200,501] \times[-1201,1201]$.

Figure 17: $p=2, \quad a_{1}=1, a_{2}=\left(a_{0}-1\right), \quad a, b=0.5$, $s=0.9, m=0.7, A=[-0.15,0.7] \times[-0.4,0.4]$.

Figure 18: $p=2, \quad a_{1}=2 / 3, a_{2}=1, \quad a, b, s, m=0.9$, $A=[-0.001,0.002] \times[-0.001,0.001]$.

Some Mandelbrot sets for cubic complex polynomial are presented here. In Figures 19-24, the graphs for cubic Mandelbrot sets are analyzed in JSOmhh. Figures 21 and 24 demonstrate the usual cubic while Figure 23 reflects the quadratic Mandelbrot sets for cubic complex polynomial. 


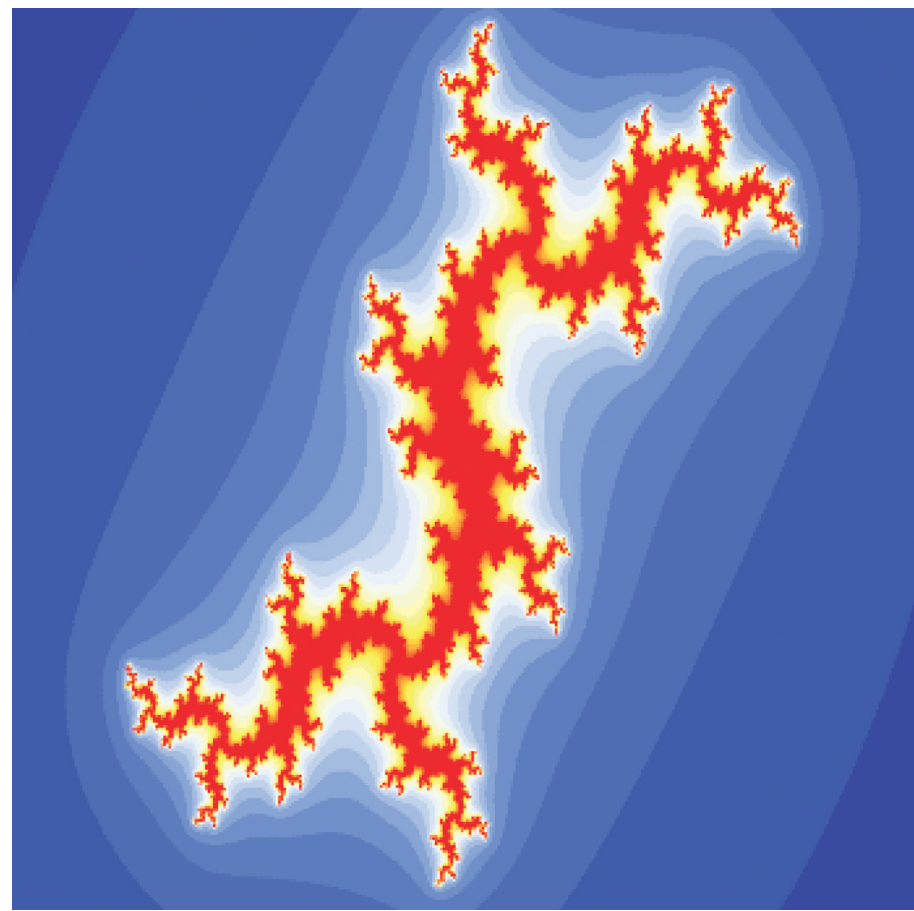

FIgURE 6: Quadratic Julia set in JSOmhh.



Figure 7: Cubic Julia set in JSOmhh.

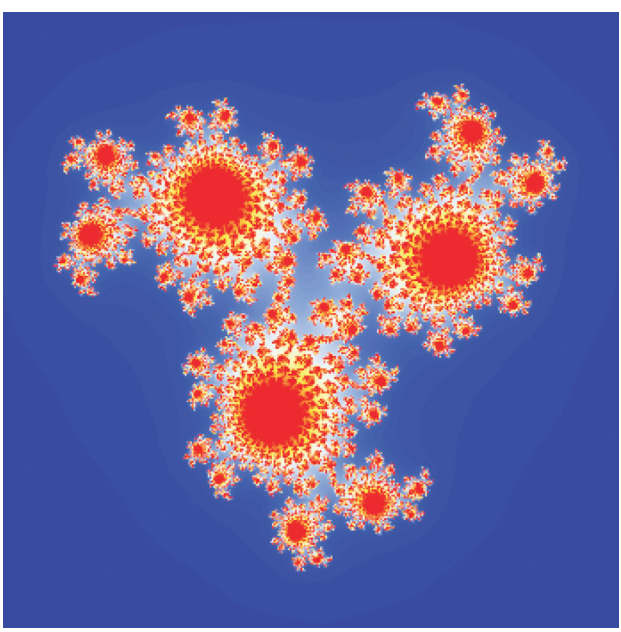

Figure 8: Cubic Julia set in JSOmhh. 


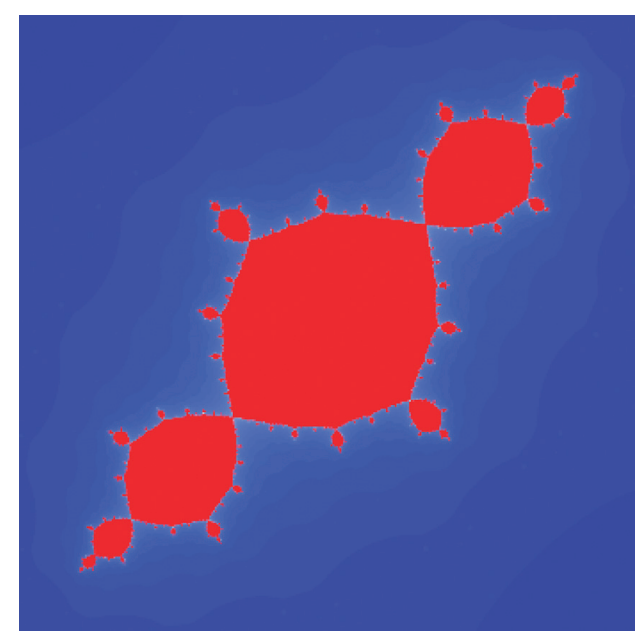

FIgure 9: Cubic Julia set in JSOmhh.

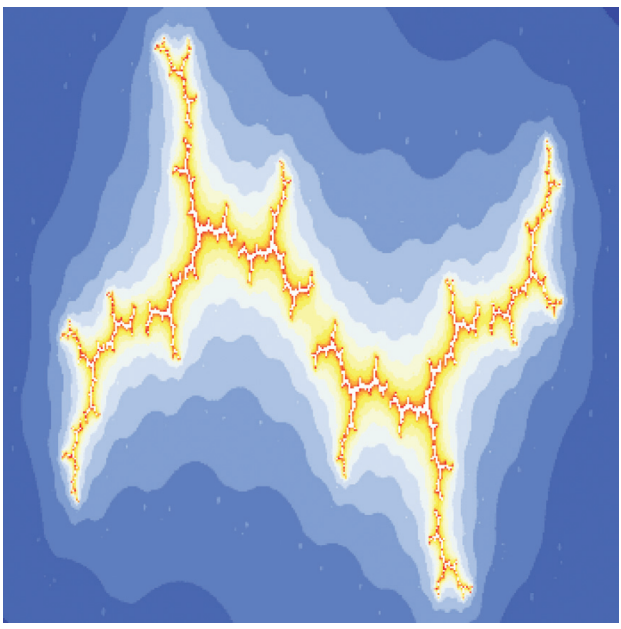

FIgURE 10: Cubic Julia set in JSOmhh.

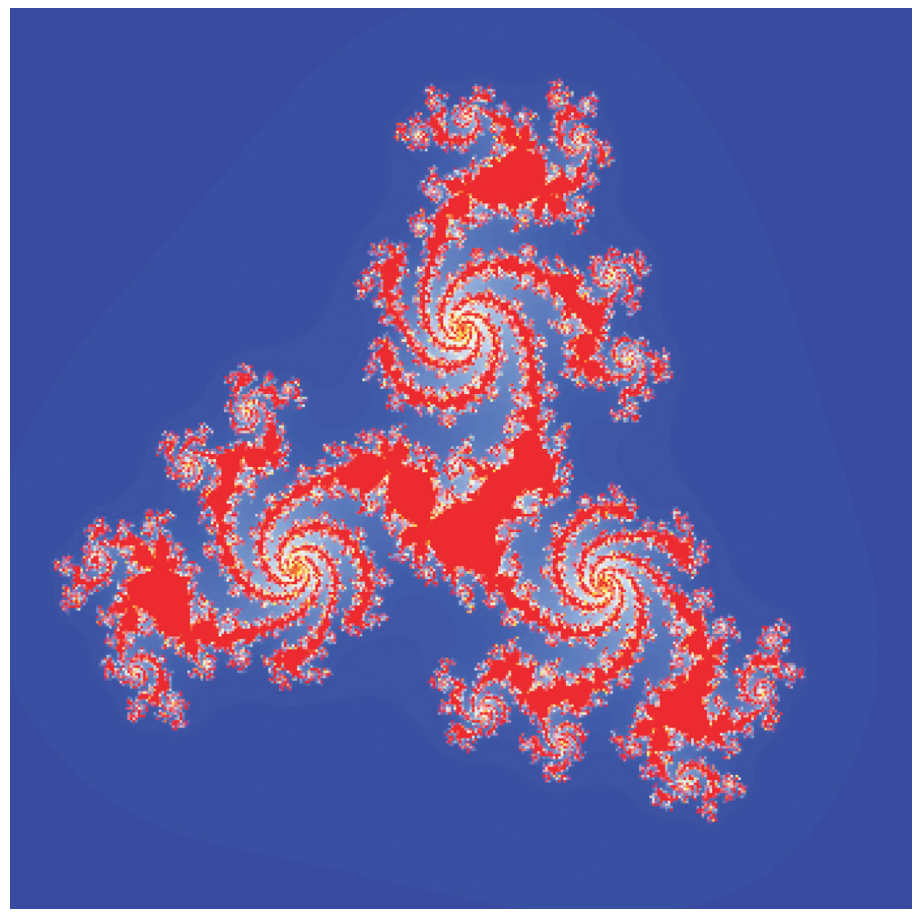

FIGURE 11: Cubic Julia set in JSOmhh. 


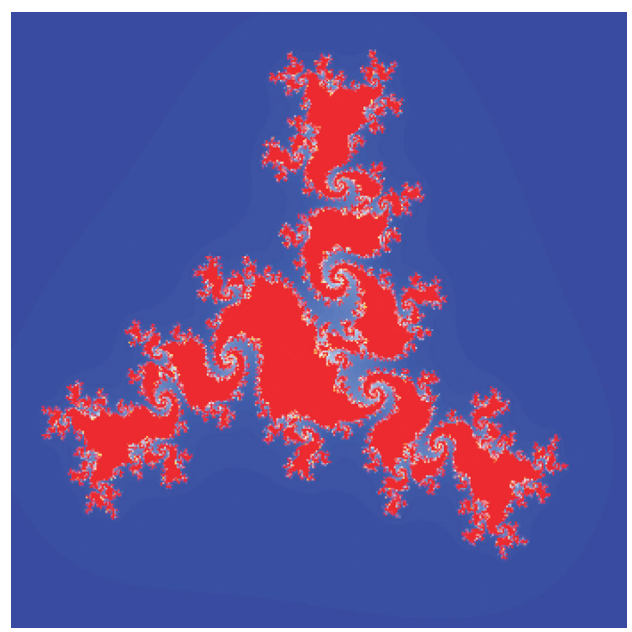

FIgURE 12: Cubic Julia set in JSOmhh.

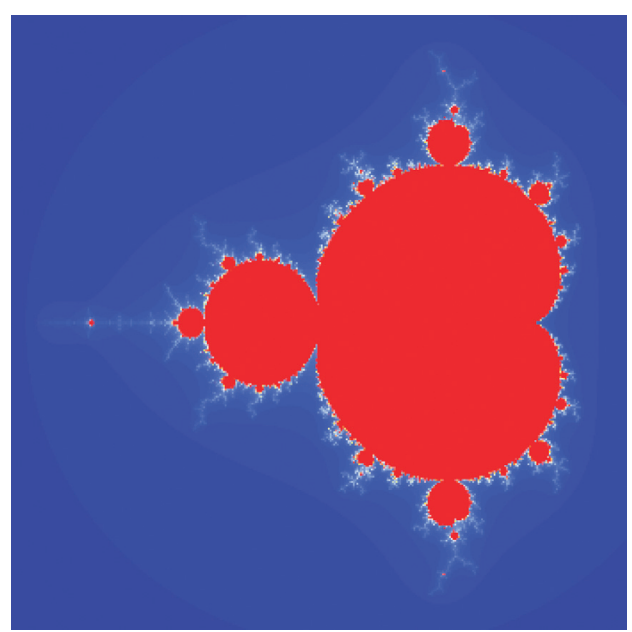

FIgUre 13: Quadratic Mandelbrot set in JSOmhh.

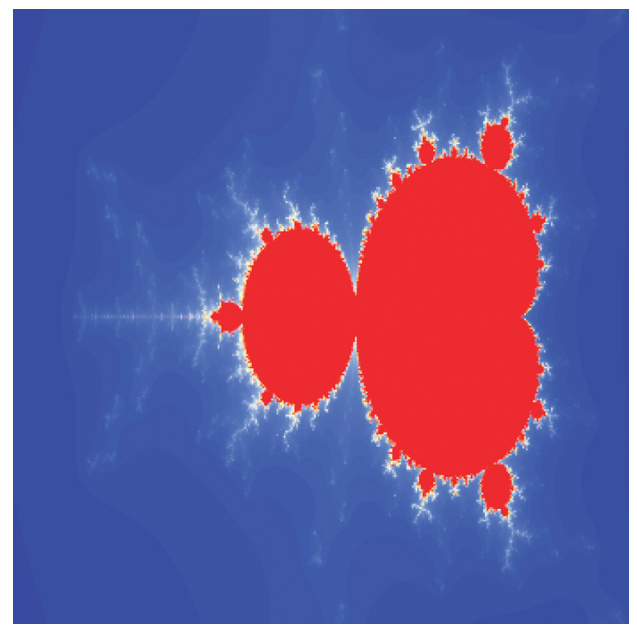

FIgURE 14: Quadratic Mandelbrot set in JSOmhh.

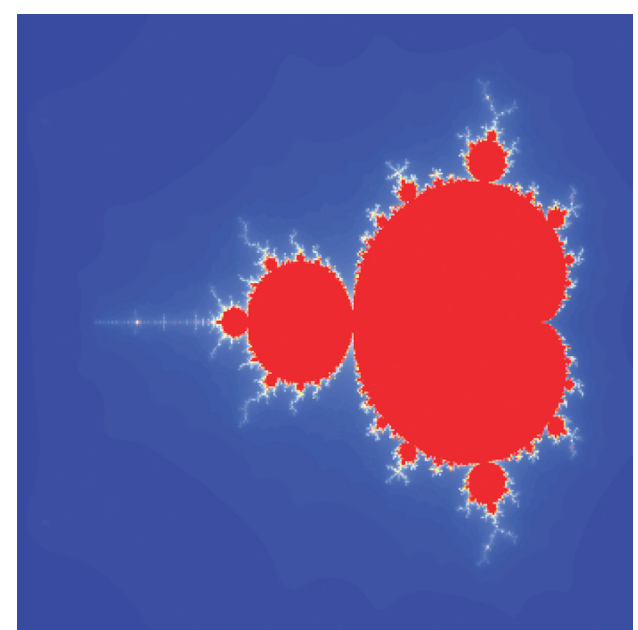

Figure 15: Quadratic Mandelbrot set in JSOmhh.



Figure 16: Quadratic Mandelbrot set in JSOmhh.

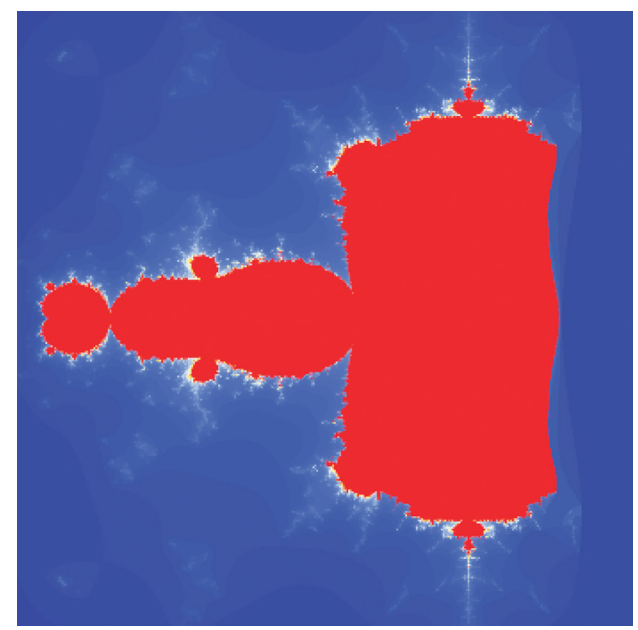

Figure 17: Quadratic Mandelbrot set in JSOmhh. 


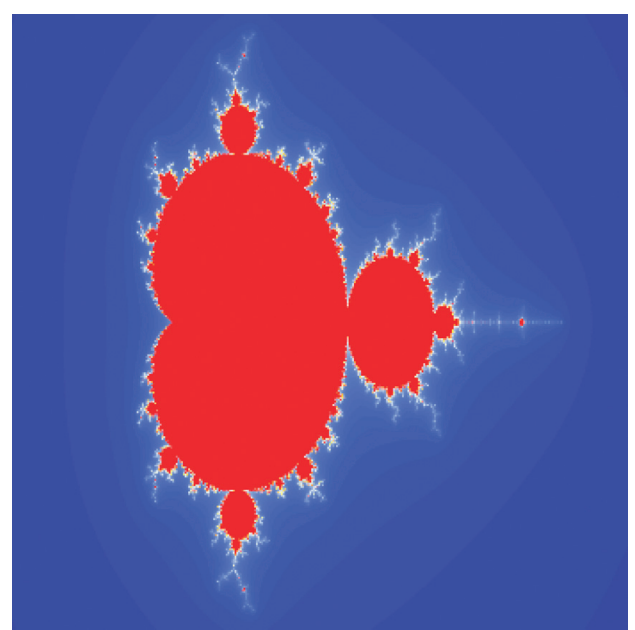

FIgURE 18: Quadratic Mandelbrot set in JSOmhh.

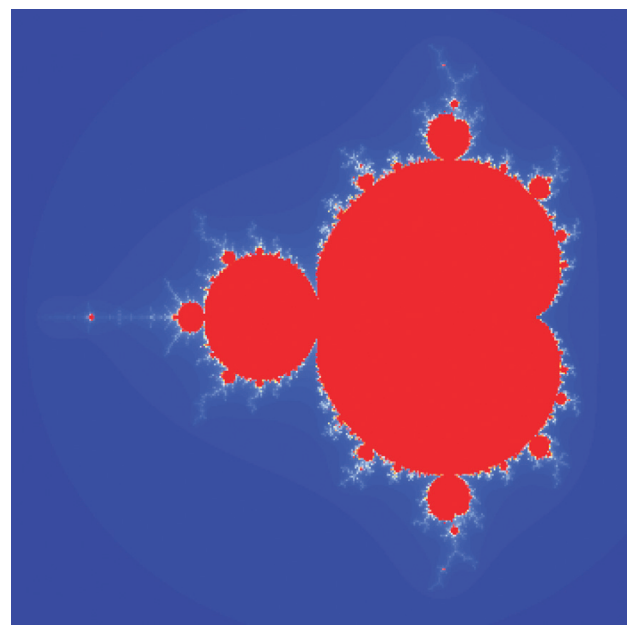

Figure 19: Cubic Mandelbrot set in JSOmhh.

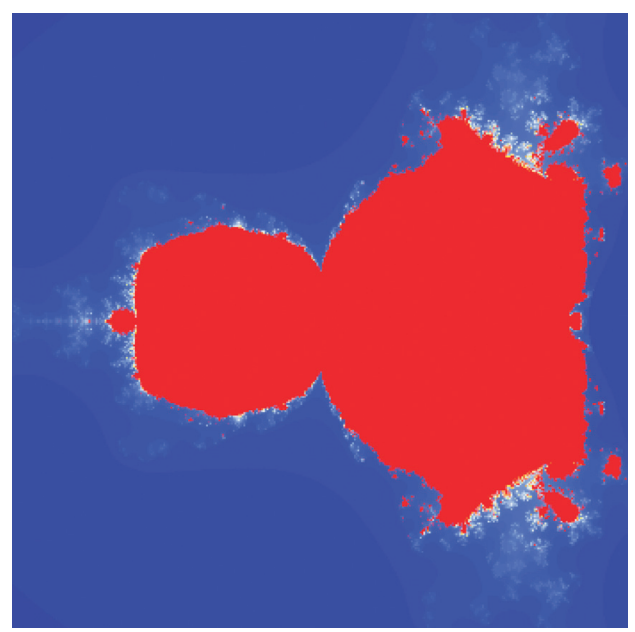

FIgure 20: Cubic Mandelbrot set in JSOmhh.

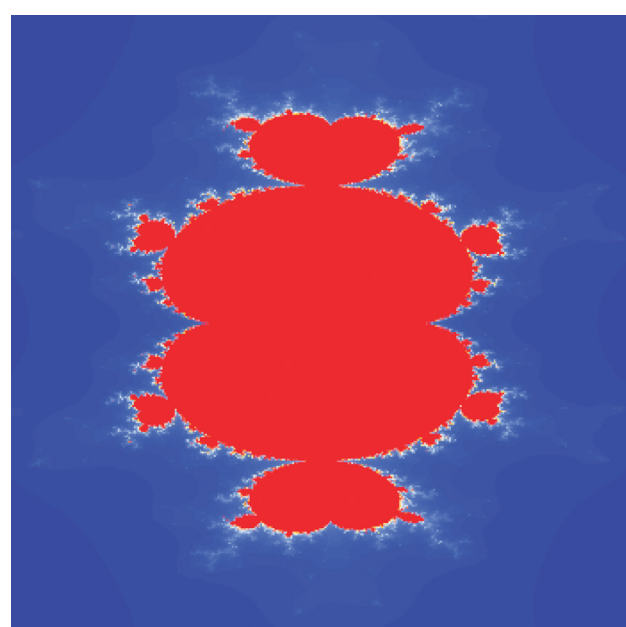

FIgure 21: Cubic Mandelbrot set in JSOmhh.

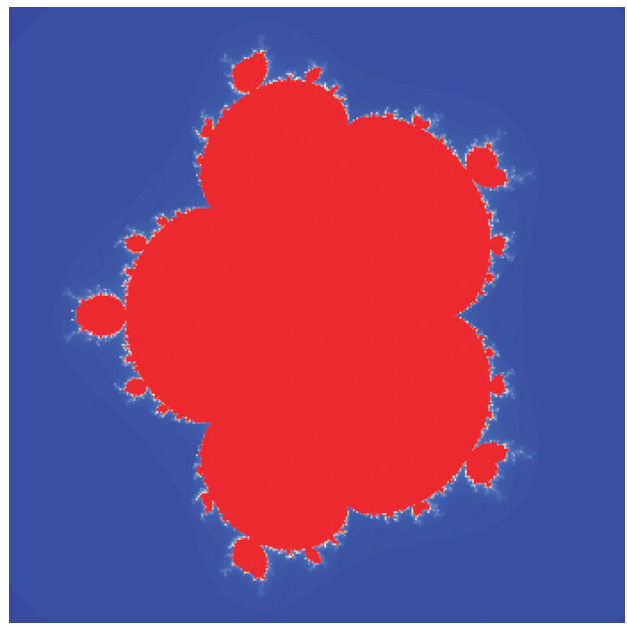

Figure 22: Cubic Mandelbrot set in JSOmhh.



FIGURE 23: Quadratic Biomorph generated in Jungck-S orbit with $s$-convexity. 


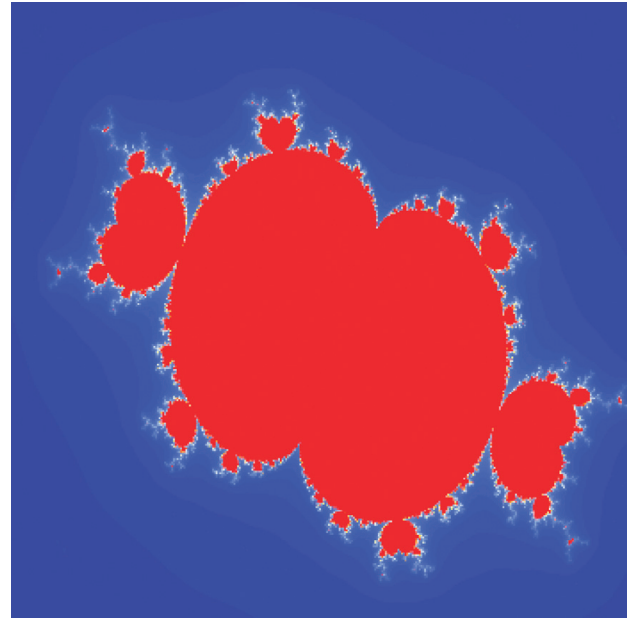

FIGURE 24: Cubic Mandelbrot set in JSOmhh.

Figure 19 is multishaped, Figure 20 is like semiquadratic semicubic, and Figure 23 is like Hexic Mandelbrot sets, respectively. The involved parameters were as follows:

Figure 19: $p=3, \quad a_{1}=2 / 3, \quad a_{2}=1, a_{3}=3, a, b, s$, $m=0.9, A=[-0.001,0.0045] \times[-0.0015,0.0015]$.

Figure 20: $p=3, a_{1}=2, a_{2}=1, a_{3}=3\left(a_{0}-1\right), a, b$, $m, s=0.5, A=[-0.9,1.05] \times[-0.9,0.9]$.

Figure 21: $p=3, a_{1}=1, a_{2}=1, a_{3}=30, a, b=0.9$, $m, s=0.5, A=[-0.03,0.025] \times[-0.045,0.045]$.

Figure 22: $p=3, a_{1}=1 / 2, a_{2}=3 a_{0}^{2}, a_{3}=3 a_{0}^{3}, a, b$, $m=0.9, s=0.5, A=[-0.2,0.2] \times[-0.2,0.2]$.

Figure 23: $p=3, a_{1}=1+\mathbf{i}, a_{2}=45, a_{3}=50, a, b, s$, $m=0.9, A=[-0.0001,0.0001] \times[-0.0002,0.0001]$.

Figure 24: $p=3, a_{1}=1+\mathbf{i}, a_{2}=1, a_{3}=50, a, b, s$, $m=0.9, A=[-0.0022,0.0017] \times[-0.0015,0.0015]$.

\section{Conclusions}

Fractals can be used to capture images of these complex structures. In addition, fractals are used to predict or analyze various biological processes or phenomena such as the growth pattern of bacteria and the pattern of situations such as nerve dendrites. We established the Jungck-S orbit with $\left(m, h_{1}, h_{2}\right)$-convexity and derived the new escape criteria for the execution of fractals. We introduced two algorithms in this paper for Julia and Mandelbrot sets. Some examples of quadratic and cubic fractals (i.e., Julia and Mandelbrot sets) were presented in detail. We observed the following characteristics of fractals in JSOmhh:

A very small change in the involved parameters caused the drastic change in fractals.

For large values of $a_{i}$ 's, area of the image will be small.

Self-similarity is observed in each image of Julia and Mandelbrot sets. Hence, the visualized complex graphs were the fractals.

In our future research work, we intend to obtain the escape radius for other Jungck-type iterative schemes with
( $\left.m, h_{1}, h_{2}\right)$-convexity. We believe this paper will attract researchers who work on the investigations of different types of fractals $[23,24]$.

\section{Data Availability}

All data required for this paper are included within this paper.

\section{Conflicts of Interest}

The authors declare that there are no conflicts of interest.

\section{Authors' Contributions}

Zhihua Chen wrote the applications of results in Engineering, enhanced the introduction, and improved the English language. Muhammad Tanveer used the software to generate fractals. Waqas Nazeer proved the main results. Jing $\mathrm{Wu}$ wrote the final draft of the paper.

\section{Acknowledgments}

The authors are thankful to Professor Yu-Ming Chu for his valuable suggestions and help in witting this paper. This work was supported by the Chinese National Natural Science Foundation under Grant 61876047. This work was sponsored in part by (1) National Natural Science Foundation of China (11501436), (2) Scientific Research Project of Shanxi Provincial Education Department (20JK0963), and (3) Xijing College Fund Project (XJ200102).

\section{References}

[1] B. B. Mandelbrot, The fractal geometry of nature, W. H. Freeman, New York, NY, 1982.

[2] A. Lakhtakia, V. V. Varadan, R. Messier, and V. K. Varadan, "On the symmetries of the Julia sets for the process $\mathrm{z} \Rightarrow \mathrm{zp}+\mathrm{c}$," Journal of Physics A: Mathematical and General, vol. 20, no. 11, pp. 3533-3535, 1987.

[3] P. Blanchard, R. L. Devaney, A. Garijo, and E. D. Russell, "A generalized version of the mcmullen domain," International Journal of Bifurcation and Chaos, vol. 18, no. 08, pp. 23092318, 2008.

[4] T. Kim, "Quaternion julia set shape optimization," Computer Graphics Forum, vol. 34, pp. 167-176, 2015.

[5] V. Drakopoulos, N. Mimikou, and T. Theoharis, "An overview of parallel visualisation methods for mandelbrot and julia sets," Computers \& Graphics, vol. 27, no. 4, pp. 635-646, 2003.

[6] Y. Sun, L. Chen, R. Xu, and R. Kong, "An image encryption algorithm utilizing julia sets and hilbert curves," PloS One, vol. 9, no. 1, p. 655, Article ID e84655, 2014.

[7] M. Rani and V. Kumar, "Superior julia set," Research in Mathematical Education, vol. 8, no. 4, pp. 261-277, 2004.

[8] M. Rani and V. Kumar, "Superior mandelbrot set," Research in Mathematical Education, vol. 8, no. 4, pp. 279-291, 2004.

[9] M. Rani and R. Agarwal, "Effect of stochastic noise on superior julia sets," Journal of Mathematical Imaging and Vision, vol. 36, no. 1, p. 63, 2010. 
[10] A. Nagi, M. Rani, and R. Chugh, "Julia sets and mandelbrot sets in noor orbit," Applied Mathematics and Computation, vol. 228, pp. 615-631, 2014.

[11] S. M. Kang, A. Rafiq, A. Latif, A. A. Shahid, and Y. C. Kwun, "Tricorns and multicorns of-iteration scheme," Journal of Function Spaces, vol. 2015, Article ID 417167, 7 pages, 2015.

[12] K. Goyal and B. Prasad, "Dynamics of iterative schemes for quadratic polynomial," AIP Conference, vol. 9, no. 2, pp. 149-153, 2001.

[13] New Julia and Mandelbrot Sets for a New Faster Iterative Process.

[14] M. F. Barnsley, Fractals Everywhere, Academic Press, Boston, Massachusetts, 2 edition, 1993.

[15] R. L. Devaney, A First Course in Chaotic Dynamical Systems: Theory and Experiment, Addison-Wesley, New York, NY, 1992.

[16] L. Xiangdong, Z. Zhiliang, W. Guangxing, and Z. Weiyong, "Composed accelerated escape time algorithm to construct the general Mandelbrot set," Fractals, vol. 9, no. 2, pp. 149$153,2001$.

[17] V. Valery, S. A. Smirnov, S. E. Korepanov, and V. Alexey, Object distance estimation algorithm for real-time fpga-based stereoscopic vision system," High-Performance Computing in Geoscience and Remote Sensing VIII, vol. 10792, Article ID 107920A, 2018.

[18] J. Barrallo, Jones, Coloring algorithms for dynamical systems in the complex plane," Visual Mathematics, vol. 1, 1999.

[19] O. Khatib, Real-time obstacle avoidance for manipulators and mobile robots Autonomous Robot Vehicles, pp. 396-404, Springer, Berlin, Germany, 1986.

[20] N. Hussain, V. Kumar, and M. A. Kutbi, "On rate of convergence of Jungck-type iterative schemes," Abstract and Applied Analysis, vol. 2013, Article ID 132626, 15 pages, 2013.

[21] W. Nazeer, S. M. Kang, M. Tanveer, and A. A. Shahid, "Fixed point results in the generation of julia and mandelbrot sets," Journal of Inequalities and Applications, vol. 2015, no. 1, p. 298, 2015.

[22] Y. C. Kwun, M. Tanveer, W. Nazeer, M. Abas, and S. M. Kang, "Fractal generation in modified jungck-s orbit," IEEE Access, vol. 7, pp. 35060-35071, 2019.

[23] D. Kaur, P. Agarwal, M. Rakshit, and M. Chand, "Fractional calculus involving $(p, q)$-mathieu type series," Applied Mathematics and Nonlinear Sciences, vol. 5, no. 2, pp. 15-34, 2020.

[24] K. A. Touchent, Z. Hammouch, and T. Mekkaoui, "A modified invariant subspace method for solving partial differential equations with non-singular kernel fractional derivatives," Applied Mathematics and Nonlinear Sciences, vol. 5, no. 2, pp. 35-48, 2020. 